OPEN ACCESS

Edited by:

Jiehui Jiang,

Shanghai University, China

Reviewed by:

Roberta Lizio,

Institute of Research and Medical Care (IRCCS) SDN, Italy

Zhen Yuan,

University of Macau, China

*Correspondence:

Yingchun Zhang yzhang94@uh.edu

Received: 11 August 2020 Accepted: 13 January 2021 Published: 03 February 2021

Citation:

Wu Z, Peng $Y$, Hong $M$ and Zhang $Y$ (2021) Gray Matter Deterioration Pattern During Alzheimer's Disease Progression: A Regions-of-Interest Based Surface Morphometry Study. Front. Aging Neurosci. 13:593898. doi: 10.3389/fnagi.2021.593898

\section{Gray Matter Deterioration Pattern During Alzheimer's Disease Progression: A Regions-of-Interest Based Surface Morphometry Study}

\author{
Zhanxiong $W^{1,2}$, Yun Peng ${ }^{2}$, Ming Hong ${ }^{1}$ and Yingchun Zhang ${ }^{2 \star}$ \\ ${ }^{1}$ School of Electronic Information, Hangzhou Dianzi University, Hangzhou, China, ${ }^{2}$ Department of Biomedical Engineering, \\ University of Houston, Houston, TX, United States
}

Accurate detection of the regions of Alzheimer's disease (AD) lesions is critical for early intervention to effectively slow down the progression of the disease. Although gray matter volumetric abnormalities are commonly detected in patients with mild cognition impairment $(\mathrm{MCl})$ and patients with $\mathrm{AD}$, the gray matter surface-based deterioration pattern associated with the progression of the disease from $\mathrm{MCl}$ to $\mathrm{AD}$ stages is largely unknown. To identify group differences in gray matter surface morphometry, including cortical thickness, the gyrification index (Gl), and the sulcus depth, 80 subjects from the Alzheimer's Disease Neuroimaging Initiative (ADNI) database were split into healthy controls (HCs; $N=20)$, early MCls (EMCl; $N=20)$, late $\mathrm{MCls}(\mathrm{LMCl} ; N=20)$, and ADs $(N=20)$. Regions-of-interest (ROI)-based surface morphometry was subsequently studied and compared across the four stage groups to characterize the gray matter deterioration during $A D$ progression. Co-alteration patterns (Spearman's correlation coefficient) across the whole brain were also examined. Results showed that patients with $\mathrm{MCl}$ and $\mathrm{AD}$ exhibited a significant reduction in cortical thickness $(p<0.001)$ mainly in the cingulate region (four subregions) and in the temporal (thirteen subregions), parietal (five subregions), and frontal (six subregions) lobes compared to HCs. The sulcus depth of the eight temporal, four frontal, four occipital, and eight parietal subregions were also significantly affected $(p<0.001)$ by the progression of AD. The Gl was shown to be insensitive to $A D$ progression (only three subregions were detected with a significant difference, $p<0.001)$. Moreover, Spearman's correlation analysis confirmed that the co-alteration pattern of the cortical thickness and sulcus depth indices is predominant during $A D$ progression. The findings highlight the relevance between gray matter surface morphometry and the stages of $A D$, laying the foundation for in vivo tracking of $A D$ progression. The co-alteration pattern of surface-based morphometry would improve the researchers' knowledge of the underlying pathologic mechanisms in AD.

Keywords: gray matter, surface morphometry, Alzheimer's disease, cognition impairment, magnetic resonance imaging 


\section{INTRODUCTION}

Alzheimer's disease $(\mathrm{AD})$ is a neurodegenerative disorder and the most common cause of dementia, which presumably starts with the aggregation of amyloid beta (Dicks et al., 2019). Gray matter volume reductions, a prominent $\mathrm{AD}$ feature because of neuronal loss, are considered as a close biological substrate of decline in cognitive functions. The decreases in gray matter volume can be measured by MRI. Studies have indicated gray matter abnormalities in patients with AD (Karas et al., 2004). Compared with healthy controls (HCs), patients with AD showed significantly lower global gray matter volume, lower whole brain volume, and greater ventricles (Guo et al., 2010). As the disease advances, gray matter abnormalities start to spread from the bilateral hippocampus, the amygdala, the entorhinal cortex, the posterior cingulate gyrus, and the medial thalamus to the parietal and frontal lobes (Moller et al., 2013). The symptomatic predementia stage of $\mathrm{AD}$, most commonly referred to as mild cognitive impairment (MCI), is critical to the development of predictive methods for early detection of $\mathrm{AD}$ and for further intervention programs (Li et al., 2018; Li K. et al., 2019; Ottoy et al., 2019; Wee et al., 2019). Different machine learning methods have been proposed to discriminate MCIs from HCs and ADs, based on the features extracted from structural MRI (Dimitriadis et al., 2017; Gomez-Sancho et al., 2018; Hojjati et al., 2018; Liu et al., 2020). Furthermore, recent evidence (Dicks et al., 2018; Tijms et al., 2018; Li R. et al., 2019; Wang et al., 2020) suggest that neuronal alterations in brain disorders tend to form patterns that resemble those of cerebral connectivity (coalteration patterns). Therefore, to monitor disease progression, powerful non-invasive biomarkers, such as gray matter diffusivity (Jacobs et al., 2013) and gray matter volume (Lee et al., 2016; Qian et al., 2019), as well as their co-alteration patterns across the whole brain, are necessary to identify $\mathrm{AD}$ at early $\mathrm{MCI}$ stage and to advance the diagnosis, treatment, and prevention of these disorders.

Voxel-based morphometry (VBM) has been frequently used to examine gray matter differences across the whole brain. Using VBM, gray and white matter volume reductions were simultaneously detected between HCs and ADs (Baxter et al., 2006; Guo et al., 2010; Ha et al., 2012; Beejesh et al., 2019). An $\mathrm{AD}$ progression model was proposed to provide anatomically specific predictions of disease spread over time with VBM (Phillips et al., 2018). Dicks et al. (2019) modeled the gray matter atrophy in $\mathrm{AD}$ as a function of time and aging using Mini-Mental State Examination (MMSE) and found that the association of atrophy with MMSE was weaker than those with time or age. Based on VBM, local gray matter volumes were compared between patients with late- and early-onset $\mathrm{AD}$ and older and younger control subjects (Moller et al., 2013, Wu et al., 2020), and interactions of age and diagnosis on the volumes of the hippocampus and the precuneus were assessed, suggesting that the patterns of atrophy might vary in the spectrum of $\mathrm{AD}$ (Moller et al., 2013). Besides gray matter volume, revealing cross effects between AD-related incipient lesions helps to understand the progression to $\mathrm{AD}$ from MCI. Machine learning models were trained on VBM and connectome estimates to detect accurately $\mathrm{AD}$-related neurodegeneration across the whole brain in a data-driven manner (Wang et al., 2019). Association between regional gray matter volume and two subtypes of psychotic symptoms in patients with mild AD was investigated, showing a distinct neural correlation between the paranoid and the nonpsychosis groups (Lee et al., 2016). With the VBM technique, Cauda et al. (2018) found that structural alterations in the gray matter tended to follow the network-like patterns, indicating that structural co-alterations were influenced by connectivity constraints rather than being randomly distributed. Manuello et al. (2017) have investigated gray matter co-alterations of AD and found a series of co-altered areas that include the left hippocampus, left and right amygdalae, right parahippocampal gyrus, and right temporal inferior gyrus. Based on VBM, these studies consistently showed a widespread gray matter co-atrophy pattern due to $\mathrm{AD}$. The co-alteration pattern may accelerate the development of neuronal abnormalities.

Unlike VBM, the surface-based morphometry methods can measure the cortical thickness and folding patterns, as well as the shape or curvature measures derived from brain surface meshes (Gutman et al., 2009; Lui et al., 2010). Previous studies demonstrated an increased accuracy of brain registration using brain surface meshes for spatial registration, compared to volume-based registration (Desai et al., 2005). Brain surface meshes permit new forms of analyses, such as the GI and the sulcus index, which measure surface complexity in 3D (Yotter et al., 2011) or cortical thickness (Righart et al., 2017). In addition, inflation or spherical mapping of the cortical surface mesh raises the buried sulci to the surface so that the mapped functional activity in these regions can be made visible. However, few studies have attempted to monitor gray matter alterations associated with MCIs and ADs using regions-of-interest (ROI)based surface morphometry based on brain surface meshing. In this study, we investigated ROI-based surface morphometry of gray matter in different stages of $\mathrm{AD}$, including $\mathrm{HC}$, EMCI, $\mathrm{LMCI}$, and $\mathrm{AD}$, aiming to identify characteristic gray matter alteration patterns in terms of cortical thickness, GI, and the sulcus depth during $\mathrm{AD}$ progression.

\section{METHODS AND MATERIALS}

\section{Subjects}

Data used in this study were obtained from the Alzheimer's Disease Neuroimaging Initiative (ADNI) database (http://adni. loni.usc.edu). The ADNI was initially launched in 2004. The primary goal of ADNI is to identify MRI, PET, biomarkers, and genetic characteristics that would support the early detection and tracking of $\mathrm{AD}$ and improve the clinical trial design (Risacher et al., 2009; Jack et al., 2010a; Petersen et al., 2010). Scans were acquired with a 3.0-T head-only Siemens Medical Solutions MRI scanner (Erlangen, Germany). T1-weighted imaging parameters were: repetition time $=2,250 \mathrm{~ms}$, echo time $=2.6 \mathrm{~ms}$, flip angle $=9$, field of view $=256 \times 256 \mathrm{~mm}$, acquisition matrix $=256 \times$ 256 , voxel size $=1 \mathrm{~mm}$ isotropic, and number of slices $=192$. The demographic data of the subjects are summarized in Table $\mathbf{1}$. The flowchart of the ROI-based surface morphometry analysis is shown in Figure 1. 
TABLE 1 | Demographics of healthy controls (HCs), mild cognition impairments (MCls), and Alzheimer's disease (ADs).

\begin{tabular}{|c|c|c|c|c|}
\hline & $\mathrm{HC}$ & Early MCls (EMCl) & Late MCls (LMCI) & AD \\
\hline Number of subjects & 20 & 20 & 20 & 20 \\
\hline Gender & $12 \mathrm{~F}: 8 \mathrm{M}$ & $8 F: 12 M$ & 9F:11M & 11F:9M \\
\hline Mean age (Std) & $73.75(4.78)$ & $75.95(7.12)$ & 74.35 (5.72) & $74.85(8.27)$ \\
\hline CDR & 0 & 0.5 & 0.5 & $0.5-1$ \\
\hline MMSE & $24-30$ & $24-30$ & $24-30$ & $20-26$ \\
\hline
\end{tabular}

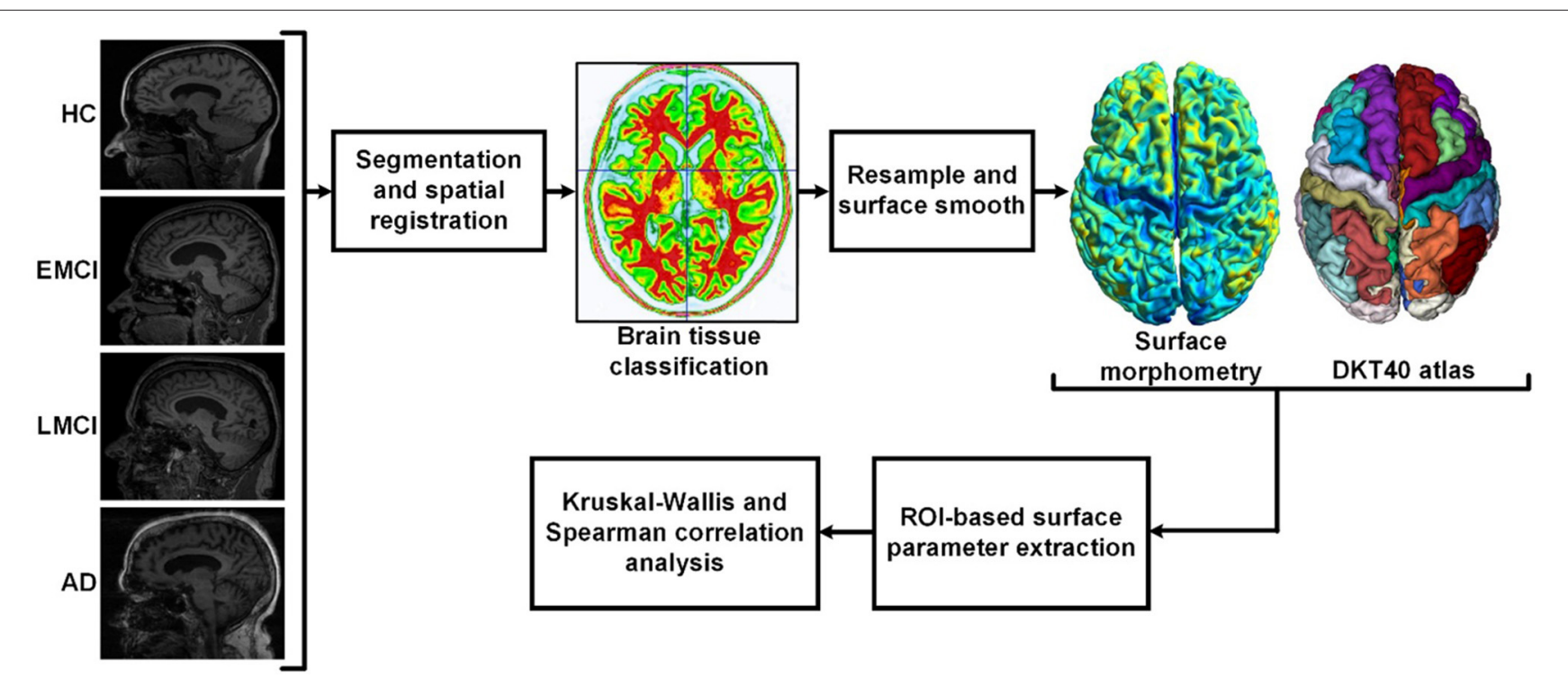

FIGURE 1 | Flowchart of the regions-of-interest (ROI)-based surface morphometry analysis. After brain extraction and segmentation (white matter, gray matter, and cerebrospinal fluid), spatial normalization was performed to correct the orientation and the size of the brain. Then, the surface of gray matter was resampled and smoothed. The ROI-based surface parameters were extracted according to the DKT40 parcellation atlas. Cortical thickness, gyrification index (GI), and sulcus depth were used to characterize the deterioration patterns of gray matter during Alzheimer's disease (AD) progression.

\section{Regions-of-Interest-Based Surface Morphometry}

T1-weighted MR image preprocessing was performed using automated procedures included in the Computational Anatomical Toolbox (CAT12), an extension to the Statistical Parametric Mapping (SPM12) package (http://www.neuro.unijena.de/cat/). First, T1-weighted images were preprocessed with intensity normalization and skull stripping, followed by the normalization of the head position along the commissural axis and the labeling of the cortical and subcortical regions. Second, the images were segmented into gray matter, white matter, and cerebrospinal fluid with the parameter of Markov random fields set to 2, and co-registered to a probabilistic brain atlas with non-linear morphing. According to the probability that a given location is of a particular tissue class (gray matter, white matter, and cerebrospinal fluid), the intensity of the image at the location, and the local spatial configuration of the location related to the labels, each MRI voxel was assigned to one specific tissue class (Dahnke et al., 2013). In this process, all T1-weighted images were spatially normalized using combinations of affine linear transformation and non-linear registration to the standard
Montreal Neurological Institute (MNI) template and segmented into gray matter, white matter, and cerebrospinal fluid. Third, a DKT40 labeling atlas was warped from standard space to subject space using the subject-specific inversed normalization parameters. All results were estimated in the native space before spatial normalization. Last, an individual brain atlas that consisted of 68 different gray matter areas was created for each participant according to the DKT40 parcellation atlas, as shown in Figure 2. The names and the corresponding indices of the parcellated regions are reported in Table 2. The pipeline used topology correction and spherical mapping to handle the partial volume effect, sulcal blurring, and asymmetry (Righart et al., 2017).

In this study, three ROI-based surface morphometry parameters were used to characterize the deterioration pattern of gray matter, including cortical thickness, the GI, and the sulcus depth:

\section{Cortical Thickness}

It is defined as the distance between the inner and the outer surface estimated from brain surface meshes, was related to 
A

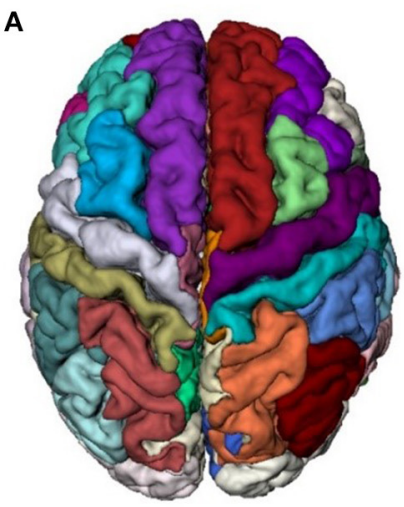

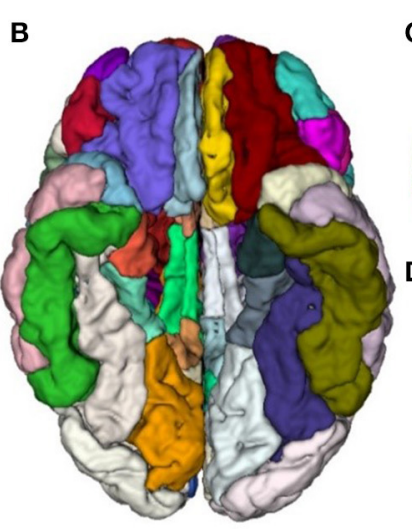

$\mathbf{C}$

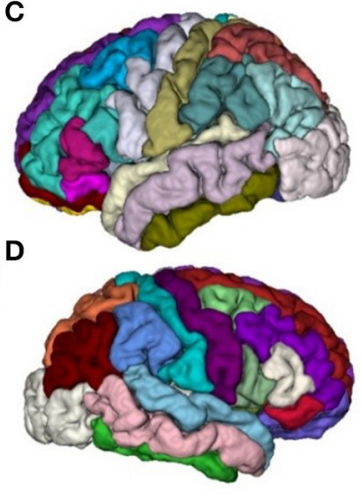

FIGURE 2 | Visualization of the DKT40 cortical parcellation atlas, comprising 68 local regions. (A) Top view, (B) Bottom view, (C) Right view, and (D) Left view.

TABLE 2 | Names and indices of the DKT40 parcellated cortical regions.

\begin{tabular}{|c|c|c|c|}
\hline Region & Region & Region & Region \\
\hline 1 bankssts_left & 2 bankssts_right & 3 caudalanteriorcingulate_left & 4 caudalanteriorcingulate_right \\
\hline 5 caudalmiddlefrontal_left & 6 caudalmiddlefrontal_right & 7 cuneus_left & 8 cuneus_right \\
\hline 9 entorhinal_left & 10 entorhinal_right & 11 fusiform_left & 12 fusiform_right \\
\hline 13 inferiorparietal_left & 14 inferiorparietal_right & 15 inferiortemporal_left & 16 inferiortemporal_right \\
\hline 17 isthmuscingulate_left & 18 isthmuscingulate_right & 19 lateraloccipital_left & 20 lateraloccipital_right \\
\hline 29 parahippocampal_left & 30 parahippocampal_right & 31 paracentral_left & 32 paracentral_right \\
\hline 33 parsopercularis_left & 34 parsopercularis_right & 35 parsorbitalis_left & 36 parsorbitalis_right \\
\hline 37 parstriangularis_left & 38 parstriangularis_right & 39 pericalcarine_left & 40 pericalcarine_right \\
\hline 41 postcentral_left & 42 postcentral_right & 43 posteriorcingulate_left & 44 posteriorcingulate_right \\
\hline 45 precentral_left & 46 precentral_right & 47 precuneus_left & 48 precuneus_right \\
\hline 61 frontalpole_left & 62 frontalpole_right & 63 temporalpole_left & 64 temporalpole_right \\
\hline 65 transversetemporal_left & 66 transversetemporal_right & 67 insula_left & 68 insula_right \\
\hline
\end{tabular}

cortical development (Dahnke et al., 2013), and identified as an important biomarker for normal development and aging (Sowell et al., 2004, 2007; Fjell et al., 2006) and pathological changes such as AD (Kuperberg et al., 2003; Sailer et al., 2003; Thompson et al., 2004; Rosas et al., 2008). Here, brain tissue segmentation was used to estimate the white matter distance and to project the local maxima (which is equal to the cortical thickness) onto other gray matter voxels using a neighboring relationship described by the white matter distance. This projection-based thickness allowed the handling of partial volume information, sulcal blurring, and sulcal asymmetries without explicit sulcus reconstruction (Dahnke et al., 2013).

\section{Gyrification Index}

It is defined as the ratio of the inner surface size to the outer surface size of an outer (usually convex) hull and was computed by averaging the absolute curvature values from each vertex of the spherical surface mesh (Luders et al., 2006).

\section{Sulcus Depth}

It is extracted based on the Euclidean distance between the central surface and its convex hull. Transformation with square root is used to render the data more normally distributed.

These surface parameters were estimated using the CAT toolbox (designed by Structural Brain Mapping Group, Departments of Psychiatry and Neurology, Jena University Hospital, Germany), which uses an internal interpolation to provide more reliable results even with low-resolution images and anisotropic spatial resolutions. Although interpolation cannot add more details to the images, the computations would benefit from the higher number of voxels, and the strip artifacts in preprocessed images are greatly reduced. While cortical thickness was estimated from the surface smoothed to $15 \mathrm{~mm}$ of full width at half maximum Gaussian kernel, GI and sulcus 


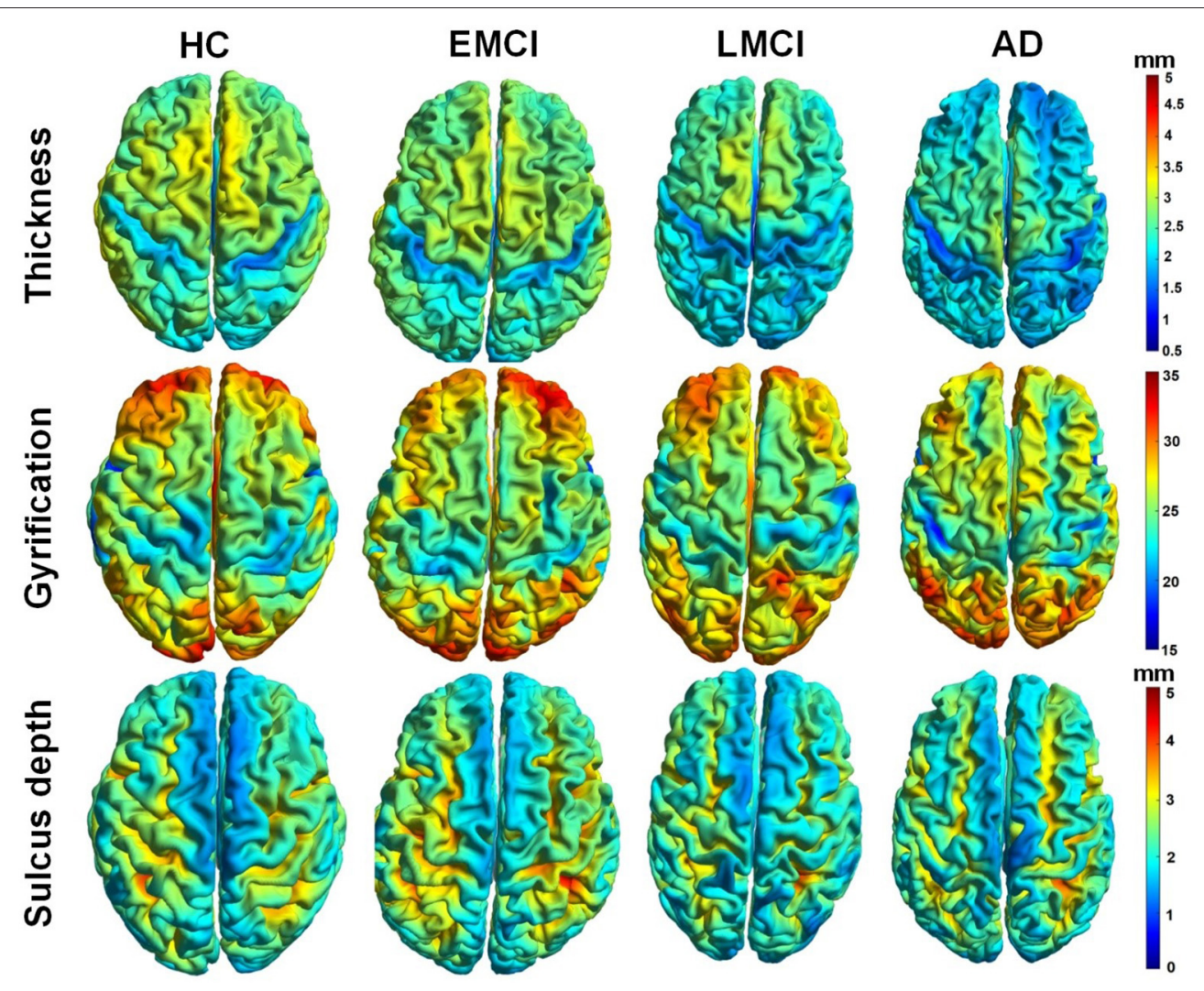

FIGURE 3 | Whole-brain mapping of surface thickness, gyrification index (GI), and sulcus depth maps estimated using CAT12 toolbox. From left to right, each column represents a subject in control, early mild cognitive impairment (EMCl), late $\mathrm{MCl}(\mathrm{LMCl})$, and $\mathrm{AD}$ groups, respectively.

depth were computed from the surface smoothed with $20 \mathrm{~mm}$ full width at half maximum (Dahnke et al., 2013).

\section{Statistical Analysis}

For each region (total 68 regions in DKT40 atlas), the gender covariate was first regressed out. Group-wise differences in cortical thickness, the GI, and the sulcus depth were assessed using the Kruskal-Wallis test. To additionally characterize the structural co-alterations in the evolution of AD, the Spearman's correlation analysis was used to investigate whether the alteration of a brain area was associated with the alteration of other brain areas. Statistical analyses were performed in MATLAB. For all analyses, significance was set at the value of $p<0.001$ (uncorrected). Effect sizes for the Kruskal-Wallis tests can be defined as the chi-squared statistic divided by $(N-1)$.

$$
\eta^{2}=\frac{\chi^{2}}{N-1}
$$

where $\chi^{2}$ is chi-squared statistic and $N$ is sample size.

\section{RESULTS}

Figure 3 demonstrated whole-brain mapping of cortical thickness, the GI, and the sulcus depth, where, from left to right, each column represents a subject in HC, EMCI, LMCI, and AD groups. Overall, across the four groups, the distributions of these parameters exhibited similarities. The greatest local cortical thickness appeared to be located in the left and right parietal lobes. The highest local gyrification was located in the frontal lobe, as well as in the occipital lobe, while the lowest GI in the left and right hemispheres appears surrounding the superior parietal gyrus and expanding into the inferior temporal gyrus. The least sulcus depth was detected in the elongated regions along the longitudinal fissure between the left and right hemispheres. As demonstrated in Figure 3, there were some differences in these surface complexity parameters across these groups, especially in cortical thickness (first row in Figure 3). Subsequently, the Kruskal-Wallis and Spearman's correlation tests were used to assess regional differences in these surface morphometry parameters across the groups.

Figures 4-6 show the nodal distribution (mean $\pm \mathrm{SD}$ ) of cortical thickness, GI, and sulcus depth for each group. After the ROI-based surface complexity was estimated according to the DKT40 atlas, the Kruskal-Wallis test was repeated for 68 regions and the regions that could be significantly identified across four groups were provided in Table 3. As reported in this table, statistically significant differences $(p<0.001$, uncorrected) in the regions, namely temporal lobe: $1,9,10,11,15,16,27,28$, $57,58,63,64$, and 67; frontal lobe: $22,26,33,34,51$, and 52; 
A
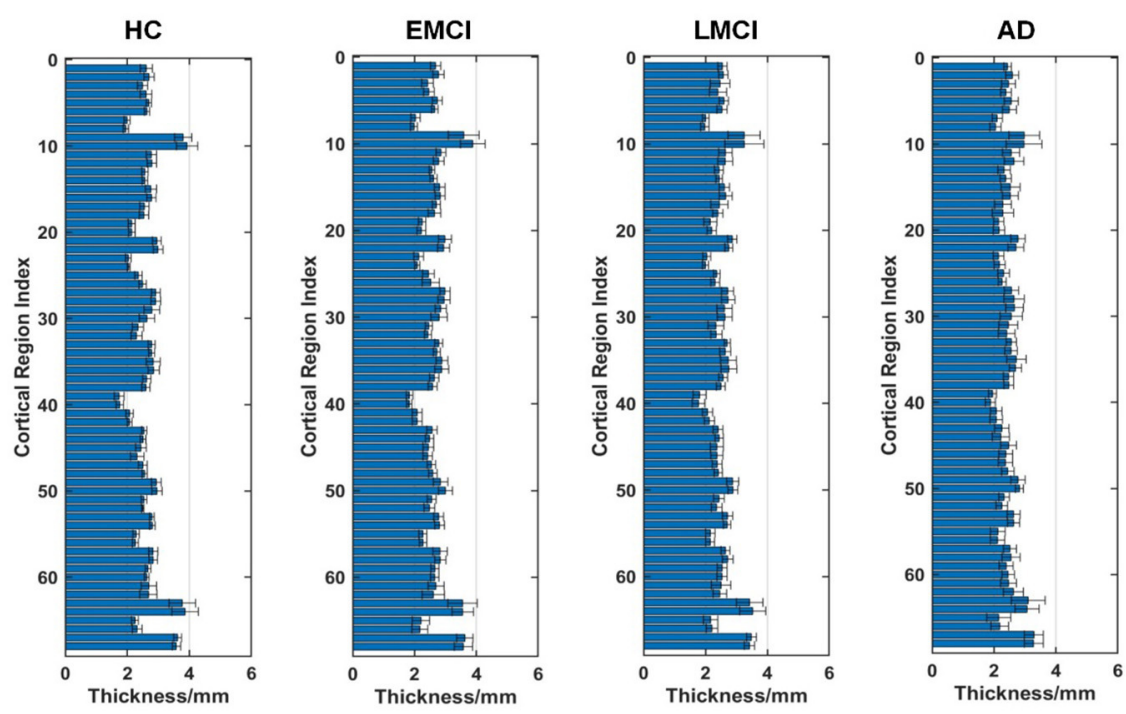

B
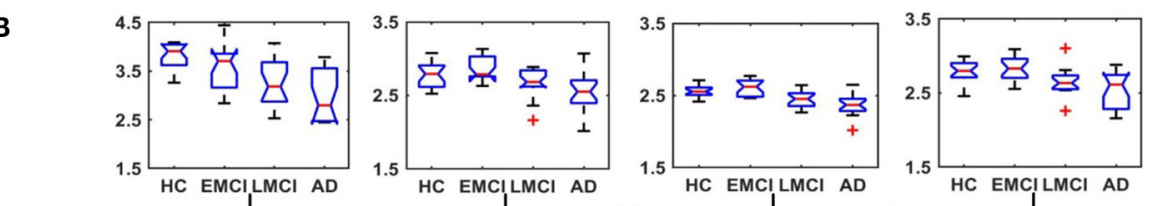
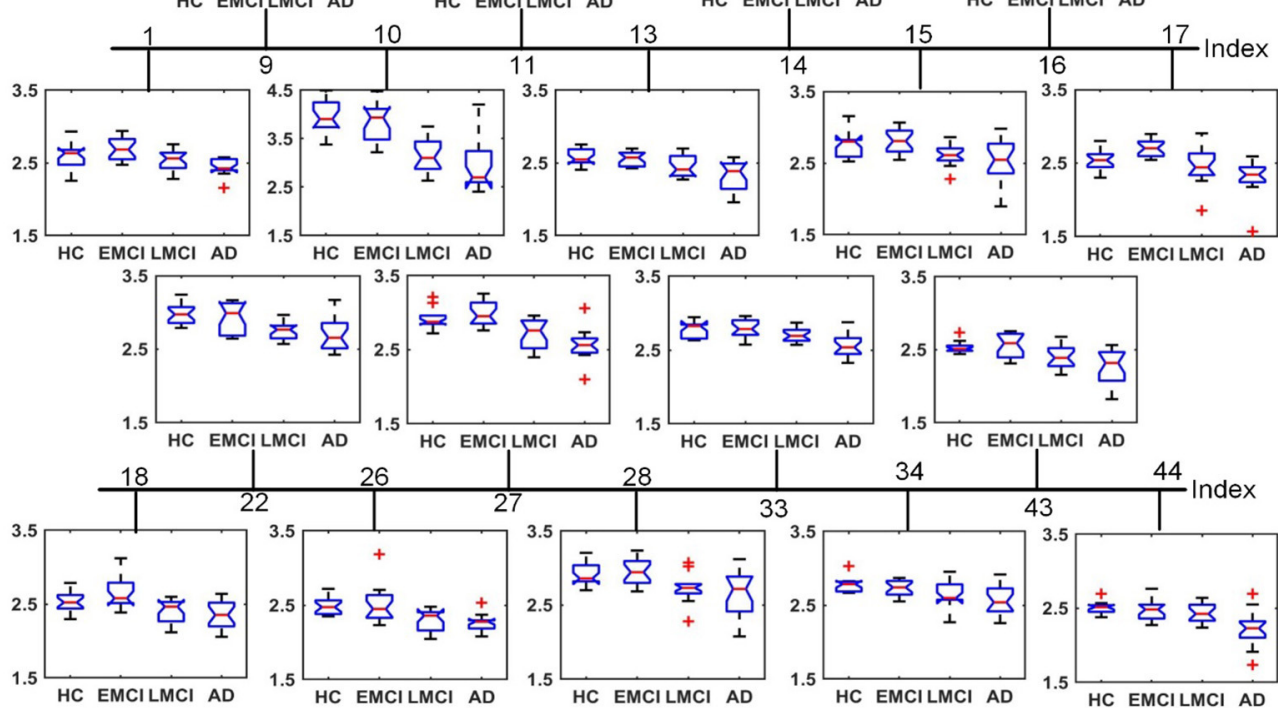

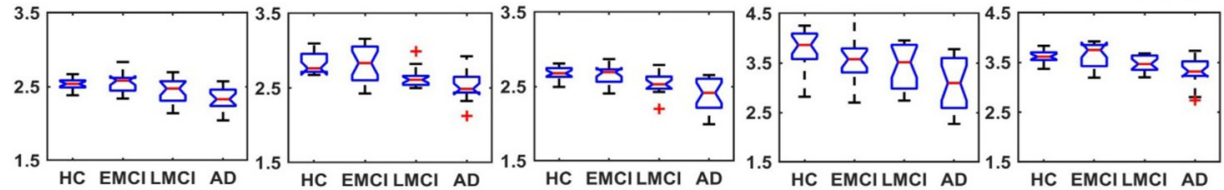

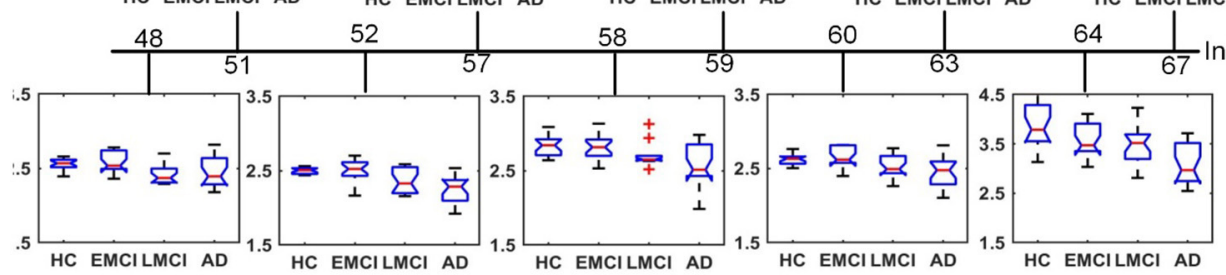

FIGURE 4 | Comparison of cortical thickness across HC, EMCI, LMCI, and AD groups. (A) The nodal distribution (mean \pm SD) of cortical thickness for each group. (B) The Kruskal-Wallis test was performed, and ROls that exhibit significant difference across four groups were listed. The value of $p$ of the Kruskal-Wallis test is reported in Table 3. Region indexes refer to Table 2. Red crosses denote outliers. 

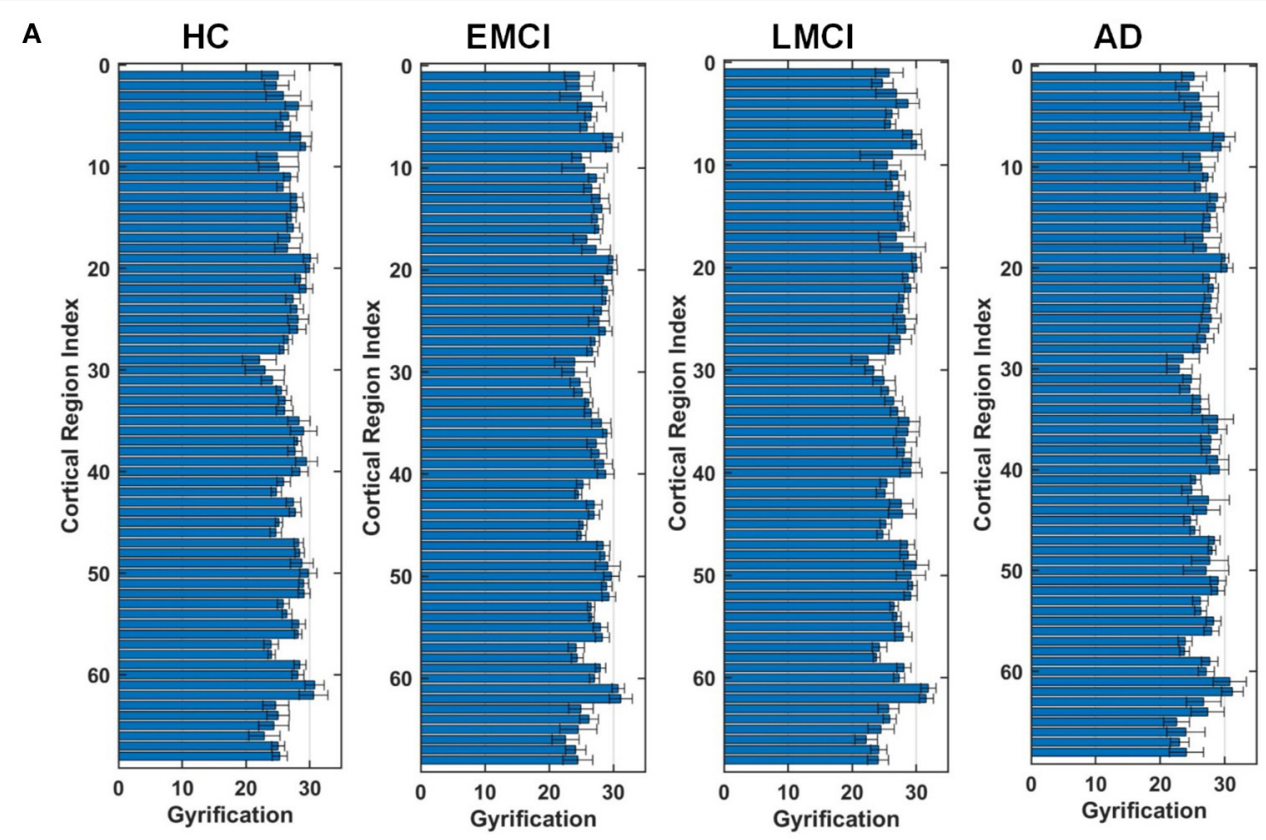

B
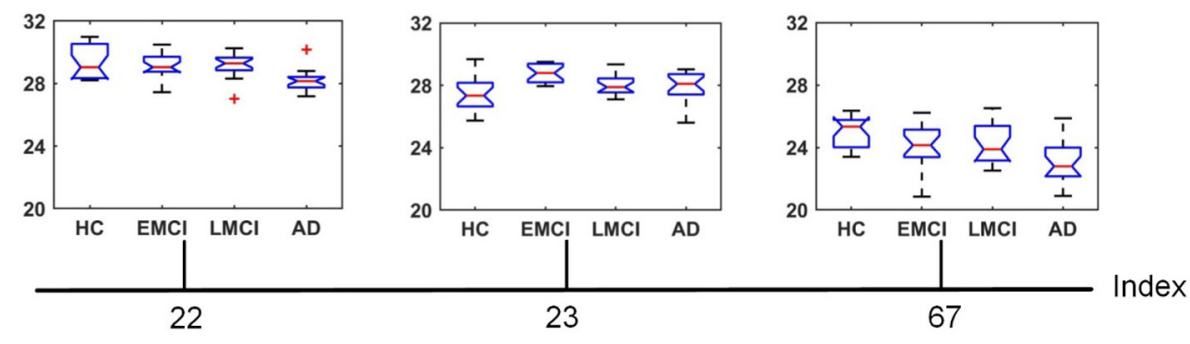

FIGURE 5 | Comparison of the Gl across $\mathrm{HC}, \mathrm{EMCl}, \mathrm{LMCl}$, and AD groups. (A) The nodal distribution (mean $\pm \mathrm{SD}$ ) of the Gl for each group. (B) The Kruskal-Wallis test was performed, and ROls that exhibit significant difference across four groups were listed. The value of $p$ of the Kruskal-Wallis test is reported in Table 3. Region indexes refer to Table 2 . Red crosses denote outliers.

parietal lobe: $13,14,48,59$, and 60 ; and cingulate: $17,18,43,44$, were found in cortical thickness. A significant difference in the GI ( $p<0.001$, uncorrected) was found only in three regions, namely 22,23 , and 67. Significant sulcus depth reductions $(p<0.001$, uncorrected) over $\mathrm{AD}$ progression were revealed, which mainly occurred in the local regions, namely temporal lobe: $2,12,16,58$, $65,66,67$, and 68; frontal lobe: 5, 6, 26, and 33; parietal lobe: 13, $14,41,45,46,55,56$, and 59; occipital lobe: $8,19,20$, and 24; and cingulate: 18 and 30 .

To reveal gray matter changes occurring simultaneously in different gray matter subregions, we characterized coalteration patterns of ROI-based surface morphometry during the evolution of AD with the Spearman's correlation analysis. Given the nodes previously designed according to the DKT40 atlas, the co-alteration matrices were constructed for cortical thickness, GI, and sulcus depth. Figure 7A shows the Spearman's correlation matrices between 68 local regions in terms of cortical thickness, GI, and sulcus depth. The matrices were binarized, and the value of +1 indicates a perfect positive correlation, i.e., the related subregions share the same decreased trend in the surface morphometric metrics. The corresponding binary networks were also illustrated in Figure 7B. Interestingly, the three co-alteration networks are different. The node degree is the number of connections that the node has with the other nodes, and it was computed to evaluate co-alteration patterns of the surface morphometric metrics over $\mathrm{AD}$ progression (Figure $7 \mathrm{C}$ ). In the co-alteration network of cortical thickness, we can find that the degrees of 26 nodes (see Figure 7C) are 25. In the sulcus depth network, the degrees of 23 nodes (see Figure 7C) are $>20$. However, for the gyrification network, the node degrees are much smaller (see Figure 7C). In accordance with the Kruskal-Wallis test, the metrics of cortical thickness and the sulcus depth are more sensitive and specific in distinguishing MCIs and ADs from HCs.

\section{DISCUSSION}

Alzheimer's disease is a progressive neurodegenerative disease characterized by a decline in memory processing and cognitive 
A

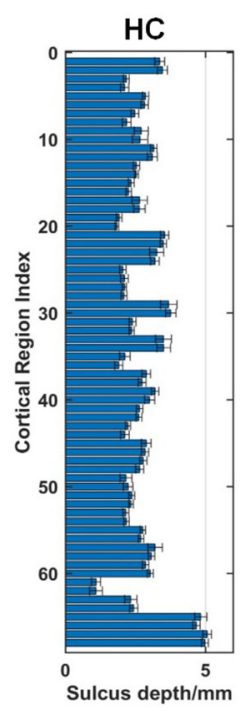

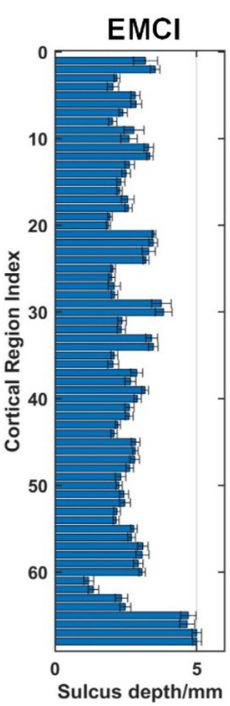
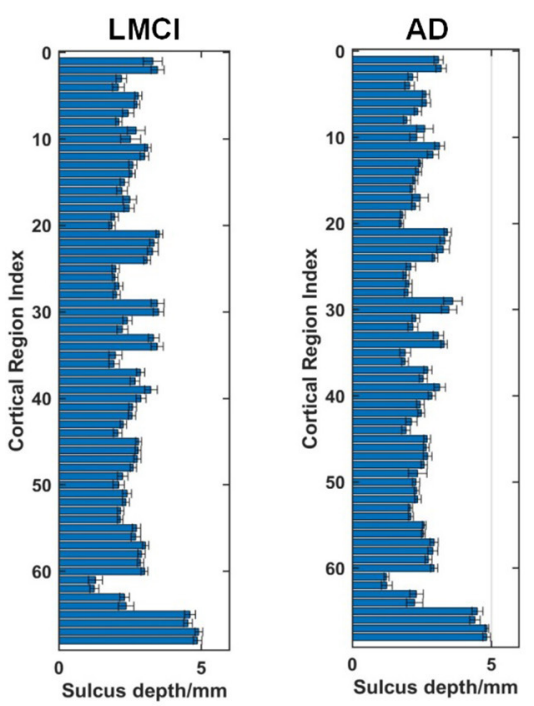

B
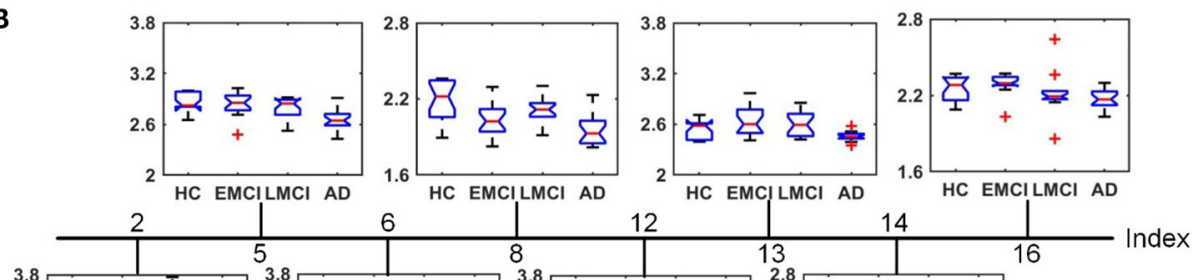

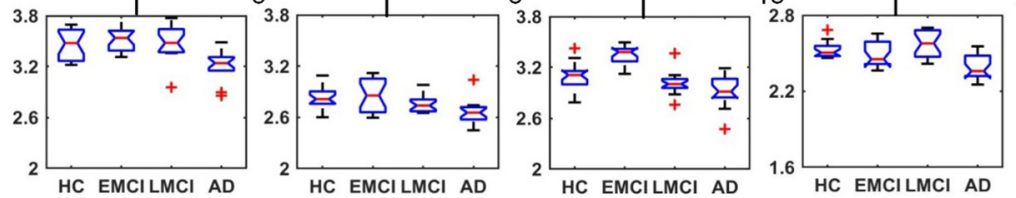
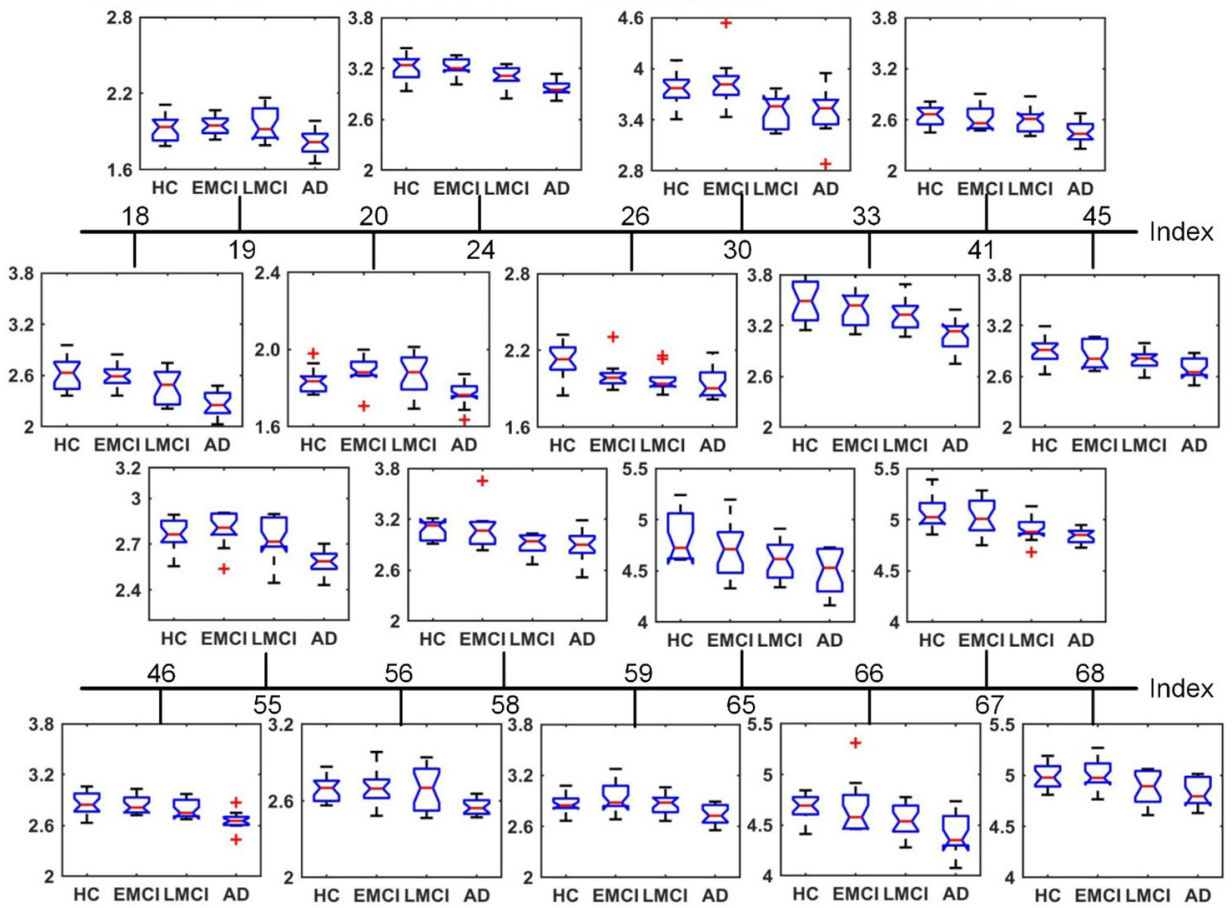

FIGURE 6 | Comparison of the sulcus depth across HC, EMCI, LMCl, and AD groups. (A) The nodal distribution (mean \pm SD) of the sulcus depth for each group. (B) The Kruskal-Wallis test was performed, and ROls that exhibit significant difference across four groups were listed. The value of $p$ of the Kruskal-Wallis test is reported in Table 3. Region indexes refer to Table 2. Red crosses denote outliers. 
function. Gray matter atrophy is considered as a close biological substrate of decline in cognitive functioning (Jack et al., 2010b). In this study, with ROI-based surface morphometry analyses based on brain surface meshes, gray matter alterations over $\mathrm{AD}$ progression were investigated. Besides cortical thickness, surface complexity (GI and sulcus depth) was estimated at a local scale, revealing a global reduction in the sulcus depth of the MCI and AD groups. The main findings of this study provide a novel perspective for understanding the pathophysiological mechanisms underlying $\mathrm{AD}$ and could potentially enhance the accuracy in the early detection and intervention of AD.

A clear deterioration pattern of gray matter over $\mathrm{AD}$ progression was shown with the Kruskal-Wallis test. Cortical thickness and sulcus depth were more pronounced during $\mathrm{AD}$ progression (Figures 4, 6), and the GI was found to be significantly different only in three local regions $(22,23,67)$ (Figure 5). This finding is broadly consistent with the findings in previous studies. Patients with AD mainly exhibited significant gray matter volume reductions in the hippocampus, the temporal lobes, the precuneus, the cingulate gyrus, the insula, and the inferior frontal cortex (Guo et al., 2010; Moller et al., 2013; Lee et al., 2016; Dicks et al., 2019). Our findings also confirmed that the brain regions exhibiting high topological centrality, considered as brain hubs, are more likely to be affected by $\mathrm{AD}$ processes, as they are located at the center of important functional networks (Cauda et al., 2018). As reported in Table 3, areas showing significant statistical decreases include insulae (67 and 68); cingulate cortices (17, 18, 43, and 44); inferior, superior, and middle temporal gyri $(15,16,27,28,57$, and 58); middle and inferior frontal $(22,26,33,34,51$, and 52); preand postcentral gyri $(13,14,41,45,46,55$, and 56). Disruption in these hub regions could impede communication between distinct gray matter regions, resulting in impaired cognitive functioning and the rapid development of $\mathrm{AD}$ abnormalities from MCI.

Evidence suggests that pathological alteration occurs long before the onset of clinical AD symptoms due to the toxic effects of amyloid-beta plaques (Chetelat et al., 2010; Johnson et al., 2014; Juan et al., 2015). In previous studies, cortical thickness changes were found to be circumscribed to the left hemisphere in patients with $\mathrm{MCI}$ and patients with $\mathrm{AD}$ using either VBM (Chetelat et al., 2002; Karas et al., 2003; Thompson et al., 2003) or the surface-based cortical thickness analysis (Lerch et al., 2005; Vivek et al., 2006). Specifically, longitudinal studies showed that the left gray matter loss of medial temporoparietal regions was strongly correlated with worse cognitive performance and that faster leftward reduction of gray matter loss was uncovered in patients with AD (Thompson et al., 2003). Our results indicated that eight regions in the left temporal lobe $(1,9,11,15,27$, 57,63 , and 67) and five regions in the right temporal lobe $(10,16,28,58$, and 64$)$ displayed a significant reduction in cortical thickness, supporting the hypothesis that AD-related cortical thickness reduction predominantly occurs in the left hemisphere. However, this spatial deterioration distribution was not observed in the parietal lobe (left: 13 and 59; right: 14, 48, and 60), the frontal lobe (left: 33 and 51; right: 22, 26, 34, and 52), and the cingulate region (left: 17 and 43; right:
TABLE 3 | The Kruskal-Wallis test on cortical thickness, GI, and sulcus depth across healthy controls $(\mathrm{HC})$, early mild cognitive impairment $(\mathrm{EMCl})$, late $\mathrm{MCl}$ (LMCl), and AD groups.

\section{Region index}

$\begin{array}{ll}\text { Cortical thickness } & 1,9,10,11,13,14,15,16,17,18,22,26,27,28,33,34, \\ & 43,44,48,51,52,57,58,59,60,63,64,67 \\ \text { Gyrification } & 22,23,67 \\ \text { Sulcus depth } & 2,5,6,8,12,13,14,16,18,19,20,24,26,30,33,41,45, \\ & 46,55,56,58,59,65,66,67,68\end{array}$

The regions associated with $p<0.001$ (uncorrected) were provided.

18 and 44). Additionally, in the statistical analysis of sulcus depth, we found that five regions in the left parietal lobe $(13,41,45,55$, and 59) and three regions (14, 46, and 56) in the right parietal lobe exhibited significant reduction. As the spatial deterioration patterns of $\mathrm{MCI}$ and $\mathrm{AD}$ may be individually different, a larger sample is needed for testing in the next step.

The pathological brains of patients with MCI and patients with $\mathrm{AD}$ are also characterized by structural co-alterations in the gray matter, which tend to follow identifiable networklike patterns (Cauda et al., 2018). The co-alteration patterns of surface morphometry parameters indicated the synchronization of gray matter deterioration between distinct gray matter parcels. Studies have revealed that gray matter co-alteration patterns of patients with $\mathrm{MCI}$ and patients with $\mathrm{AD}$ have a less optimal topological organization characterized by increased segregation and decreased integration (Yong et al., 2008; Tijms et al., 2013; Romerogarcia et al., 2016). By considering the cortical co-alteration pattern as a graph and by studying its edge strength (Spearman's correlation) features at the network level, the cross effects between AD-related incipient lesions may be disclosed. In this study, correlated changes in cortical thickness, GI, and sulcus depth were used to assess the correlation strength across the whole brain and to investigate temporal differences in cross-cortical correlations between groups. Our results provide evidence that alterations of gray matter thickness and sulcus depth are network-like distributed (Figure 7B). This co-alteration exhibits a topological structure and includes some pathological regions that have been thought to be important functional hubs of the brain. As shown in Figure 7C, the degree of these local regions (Table 3) estimated from the cortical thickness correlation matrix was between 18 and 25, except region 48 (precuneus_right), and the degree extracted from the sulcus depth correlation coefficient was between 8 and 22, except 8 (cuneus_right), 14 (inferiorparietal_right), and 19 (lateraloccipital_left). The finding confirms that the primary deterioration in some atrophic regions might lead to a secondary deterioration in other connected areas. The co-alteration patterns of brain atrophy caused by $\mathrm{AD}$ appeared to considerably resemble the patterns of brain structural connections (Cauda et al., 2018). However, from the gray matter co-alteration analyses, we still cannot identify the causal relationship between the altered gray matter parcels. It may be a chance to reveal neuropathological co-alterations patterns in patients with $\mathrm{MCI}$ 
A

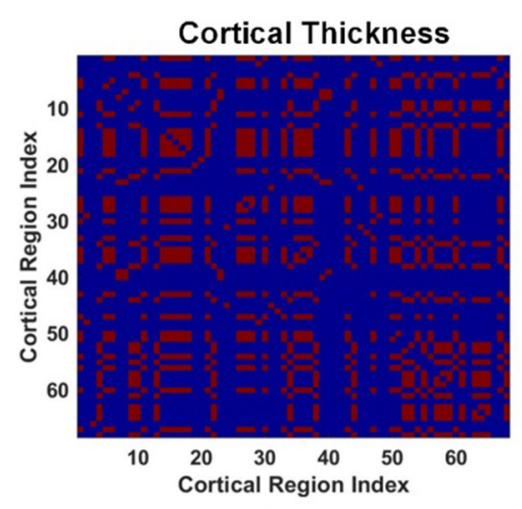

B

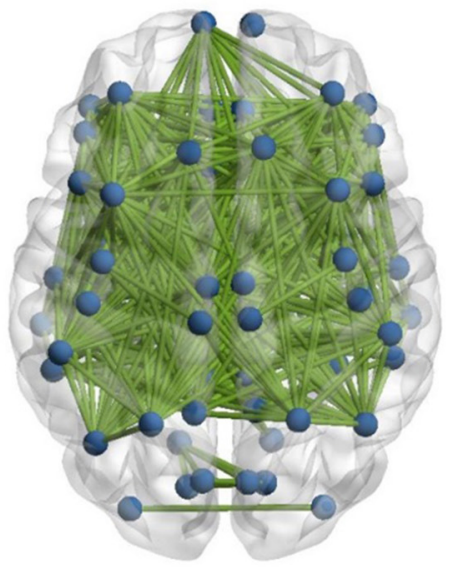

C

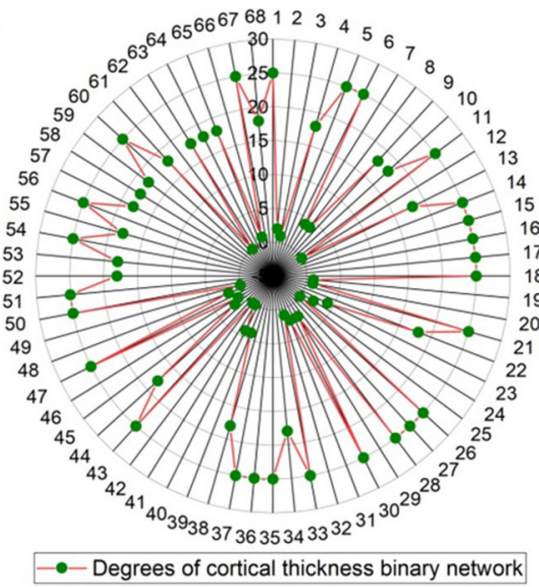

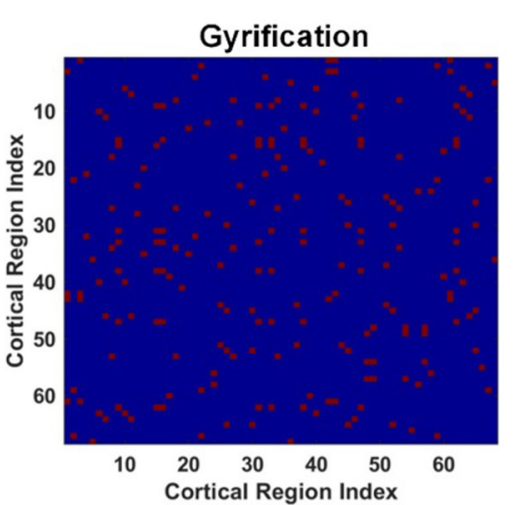
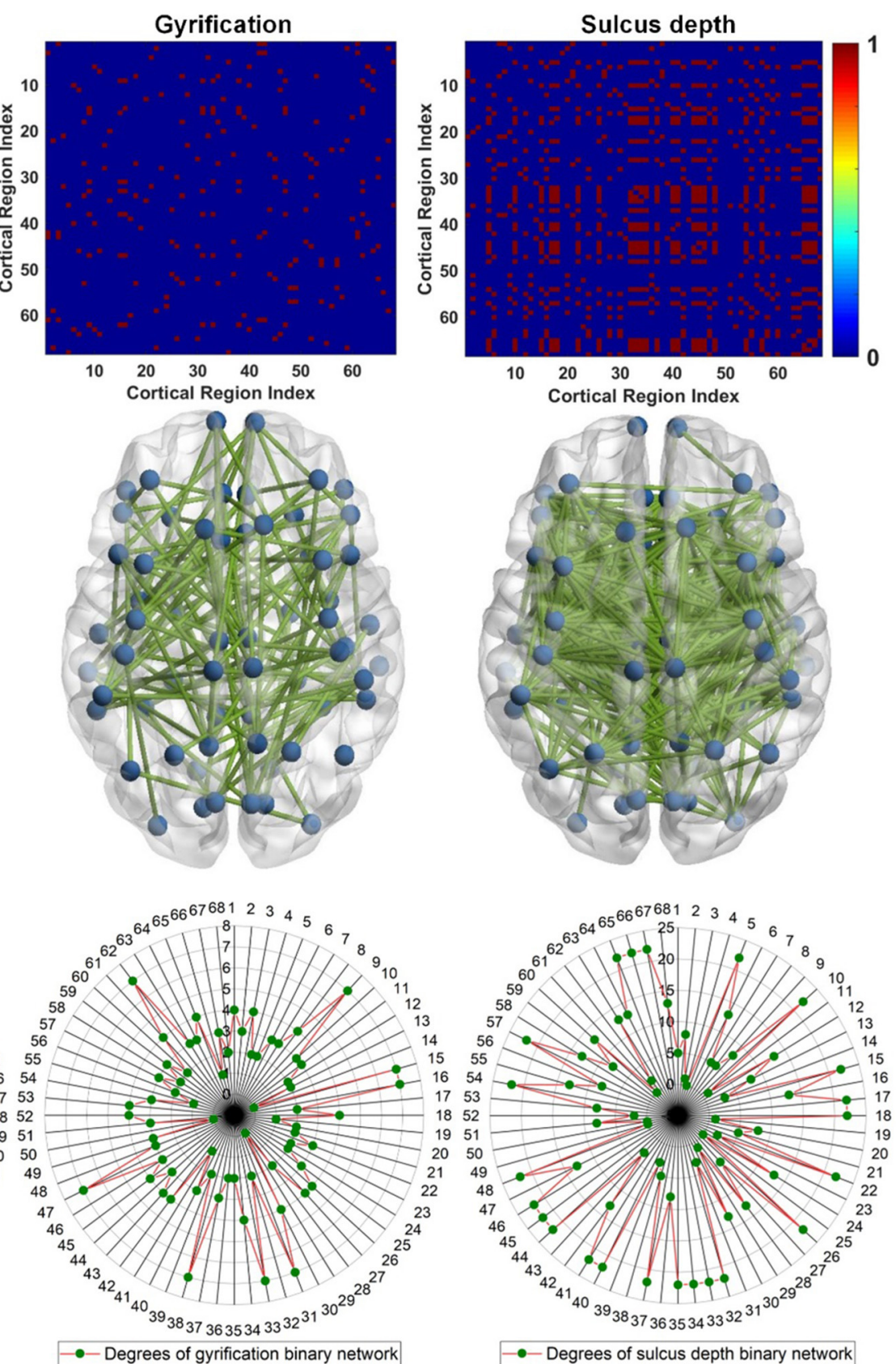

FIGURE 7 | Surface morphometric co-alteration patterns among different cortical subregions. For cortical subregion indices, please refer to Table 2. (A) Binarized Spearman's correlation matrices estimated from the ROI-based surface morphometric metrics, including cortical thickness, Gl, and sulcus depth. The correlation matrices were thresholded at the value of 1 . The value of 1 indicates that the related subregions share the same decreased trend in surface morphometry (B) Binary networks correspond to the Spearman's correlation matrices in (A). (C) Degree of each cortical subregion estimated from (A).

and patients with $\mathrm{AD}$, with a combination of functional and anatomic connectivity estimation.

A novel aspect of this study is the assessment of the ROI-based surface morphometric alteration across HC, EMCI, LMCI, and AD groups. The findings are basically in line with the literature showing the associations of gray matter volume morphometry with MCIs and ADs. This might suggest a greater sensitivity of surface estimates in detecting MCIand $\mathrm{AD}$-related neurodegeneration compared with gray matter voxel-based morphometry. However, the results in this study have several limitations to be interpreted with caution. First, this study was limited by a relatively small sample size. Although we were able to detect effects with this sample size, a larger sample would be optimal for surface morphometry 
analysis. Second, there is an increased risk for false-positive results because we used uncorrected $(p<0.001)$ thresholds for surface morphometry analysis due to our sample size. Third, brain parcellation may influence the characterization of surface morphometry during AD progression (Messe, 2019; Wu et al., 2019), which deserves further study. Last, the education information of participants and neuropsychological markers are not available in the ADNI database, so they have not been taken into account in the statistical analysis in this study. Despite these limitations, to our knowledge, this is the first report to show the association of brain regional gray matter surface complexity with $\mathrm{AD}$ progression. Further, multimodal neuroimaging studies are needed to investigate associations between regional structural brain atrophy and cognition declines in patients with $\mathrm{AD}$. More rigorous methods to combine multimodal MRI brain imaging (structural MRI, diffusion MRI, and functional MRI) may be required. Combining structural brain imaging and connectivity for in vivo tracking of $\mathrm{AD}$ related lesions in the asymptomatic stages may be a promising method, facilitating an understanding of how the co-alteration patterns found in this study were constrained by structural or functional connectivity.

\section{CONCLUSION}

This study reported the ROI-based surface morphometry of gray matter across HC, EMCI, LMCI, and AD groups and identified characteristic alteration patterns in surface morphometry during AD progression. Patients with MCI and patients with $\mathrm{AD}$ showed considerable reduction in cortical thickness and surface complexity indices. These parameters could potentially serve as biomarkers for the prediction of AD progression. Future longitudinal studies should determine whether these markers are able to detect gray matter changes with therapies aimed at slowing the disease progression. The possibility of combining structural brain imaging and anatomical or functional connectivity for in vivo tracking of AD-linked lesions in the asymptomatic stages is worth further exploration.

\section{DATA AVAILABILITY STATEMENT}

Publicly available datasets were analyzed in this study. This data can be found at: http://adni.loni.usc.edu/.

\section{ETHICS STATEMENT}

Ethical review and approval was not required for the study on human participants in accordance with the local legislation and institutional requirements. Written informed consent for participation was not required for this

\section{REFERENCES}

Baxter, L. C., Sparks, D. L., Johnson, S. C., Lenoski, B., Lopez, J. E., Connor, D. J., et al. (2006). Relationship of cognitive measures and gray and white matter in Alzheimer's disease. J. Alzheimer Dis. 9, 253-260. doi: 10.3233/JAD-2006-9304 study in accordance with the national legislation and the institutional requirements.

\section{AUTHOR CONTRIBUTIONS}

ZW: data analysis, result interpretation, manuscript drafting and revision. YP: result interpretation, manuscript drafting, and revision. MH: data analysis and manuscript drafting. YZ: data analysis, result interpretation, and manuscript revision. All authors contributed to the article and approved the submitted version.

\section{FUNDING}

The research is supported in part by the Natural Science Foundation of Zhejiang Province (LY20E070005 and LY17E070007), National Natural Science Foundation of China (51207038), China Scholarship Council, and the University of Houston. Data collection and sharing for this project was funded by the Alzheimer's Disease Neuroimaging Initiative (ADNI) (National Institutes of Health Grant No. U01AG024904) and DOD ADNI (Department of Defense award number W81XWH12-2-0012). ADNI is funded by the National Institute on Aging, the National Institute of Biomedical Imaging and Bioengineering, and through generous contributions from the following: AbbVie, Alzheimer's Association; Alzheimer's Drug Discovery Foundation; Araclon Biotech; BioClinica, Inc.; Biogen; BristolMyers Squibb Company; CereSpir, Inc.; Cogstate; Eisai Inc.; Elan Pharmaceuticals, Inc.; Eli Lilly and Company; EuroImmun; F. Hoffmann-La Roche Ltd and its affiliated company Genentech, Inc.; Fujirebio; GE Healthcare; IXICO Ltd.; Janssen Alzheimer Immunotherapy Research \& Development, LLC.; Johnson \& Johnson Pharmaceutical Research \& Development LLC.; Lumosity; Lundbeck; Merck \& Co., Inc.; Meso Scale Diagnostics, LLC.; NeuroRx Research; Neurotrack Technologies; Novartis Pharmaceuticals Corporation; Pfizer Inc.; Piramal Imaging; Servier; Takeda Pharmaceutical Company; and Transition Therapeutics. The Canadian Institutes of Health Research is providing funds to support ADNI clinical sites in Canada. Private sector contributions are facilitated by the Foundation for the National Institutes of Health (www.fnih.org). The grantee organization is the Northern California Institute for Research and Education.

\section{ACKNOWLEDGMENTS}

The study is coordinated by the Alzheimer's Therapeutic Research Institute at the University of Southern California. ADNI data are disseminated by the Laboratory for Neuro Imaging at the University of Southern California. 
anatomic and genetic connectivity. Brain 141, 3211-3232. doi: 10.1093/brain/a wy 252

Chetelat, G., Desgranges, B., De La Sayette, V., Viader, F., Eustache, F., and Baron, J. C. (2002). Mapping gray matter loss with voxel-based morphometry in mild cognitive impairment. Neuroreport 13, 1939-1943. doi: 10.1097/00001756-200210280-00022

Chetelat, G., Villemagne, V. L., Pike, K. E., Baron, J. C., Bourgeat, P., Jones, G., et al. (2010). Larger temporal volume in elderly with high versus low beta-amyloid deposition. Brain 133, 3349-3358. doi: 10.1093/brain/awq187

Dahnke, R., Yotter, R. A., and Gaser, C. (2013). Cortical thickness and central surface estimation. Neuroimage 65, 336-348. doi: 10.1016/j.neuroimage.2012.09.050

Desai, R., Liebenthal, E., Possing, E. T., Waldron, E., and Binder, J. R. (2005). Volumetric vs. surface-based alignment for localization of auditory cortex activation. Neuroimage 26, 1019-1029. doi: 10.1016/j.neuroimage.2005.03.024

Dicks, E., Tijms, B. M., Kate, M., Gouw, A. A., Benedictus, M. R., Teunissen, C. E., et al. (2018). Gray matter network measures are associated with cognitive decline in mild cognitive impairment. Neurobiol. Aging 61, 198-206. doi: 10.1016/j.neurobiolaging.2017.09.029

Dicks, E., Vermunt, L., van der Flier, W. M., Visser, P. J., Barkhof, F., Scheltens, P., et al. (2019). Modeling grey matter atrophy as a function of time, aging or cognitive decline show different anatomical patterns in Alzheimer's disease. Neuroimage Clin. 22:101786. doi: 10.1016/j.nicl.2019.101786

Dimitriadis, S. I., Liparas, D., and Tsolaki, M. N. (2017). Random forest feature selection, fusion and ensemble strategy: combining multiple morphological MRI measures to discriminate among healhy elderly, MCI, cMCI and alzheimer's disease patients: from the alzheimer's disease neuroimaging initiative (ADNI) database. J. Neurosci. Methods 302, 14-23. doi: 10.1016/j.jneumeth.2017.12.010

Fjell, A. M., Walhovd, K. B., Reinvang, I., Lundervold, A., Salat, D., Quinn, B. T., et al. (2006). Selective increase of cortical thickness in high-performing elderly-structural indices of optimal cognitive aging. Neuroimage 29, 984-994. doi: 10.1016/j.neuroimage.2005.08.007

Gomez-Sancho, M., Tohka, J., and Gomez-Verdejo, V. (2018). Comparison of feature representations in MRI-based MCI-to-AD conversion prediction. Magn. Reson. Imaging 50, 84-95. doi: 10.1016/j.mri.2018.03.003

Guo, X., Wang, Z., Li, K., Li, Z., Qi, Z., Jin, Z., et al. (2010). Voxel-based assessment of gray and white matter volumes in Alzheimer's disease. Neurosci. Lett. 468, 146-150. doi: 10.1016/j.neulet.2009.10.086

Gutman, B., Wang, Y., Morra, J., Toga, A. W., and Thompson, P. M. (2009). Disease classification with hippocampal shape invariants. Hippocampus 19, 572-578. doi: 10.1002/hipo.20627

Ha, S. Y., Youn, Y. C., Kim, S. Y., Hsiung, G. Y. R., Ahn, S. W., Shin, H. W., et al. (2012). A voxel-based morphometric study of cortical gray matter volume changes in Alzheimer's disease with white matter hyperintensities. J. Clin. Neurosci. 19, 1506-1510. doi: 10.1016/j.jocn.2011.11.041

Hojjati, S. H., Ebrahimzadeh, A., Khazaee, A., and Babajani-Feremi, A. (2018). Predicting conversion from MCI to AD by integrating rs-fMRI and structural MRI. Comput. Biol. Med. 102, 30-39. doi: 10.1016/j.compbiomed.2018.09.004

Jack, C. R., Bernstein, M. A., Fox, N. C., Paul, T., Danielle, H., Bret, B., et al. (2010a). The Alzheimer's disease neuroimaging initiative (ADNI): MRI methods. J. Magn. Reson. Imaging 27, 685-691. doi: 10.1002/jmri.21049

Jack, C. R., Knopman, D. S., Jagust, W. J., Shaw, L. M., Aisen, P. S., Weiner, M. W., et al. (2010b). Hypothetical model of dynamic biomarkers of the Alzheimer's pathological cascade. Lancet Neurol. 9, 119-128. doi: 10.1016/S1474-4422(09)70299-6

Jacobs, H. I., Van Boxtel, M. P., Gronenschild, E., Uylings, H. B., Jolles, J., and Verhey, F. R. (2013). Decreased gray matter diffusivity: a potential early Alzheimer's disease biomarker?. Alzheimers Dement. 9, 93-97. doi: 10.1016/j.jalz.2011.11.004

Johnson, S. C., Christian, B. T., Okonkwo, O. C., Oh, J. M., Harding, S., $\mathrm{Xu}, \mathrm{G}$., et al. (2014). Amyloid burden and neural function in people at risk for Alzheimer's disease. Neurobiol. Aging 35, 576-584. doi: 10.1016/j.neurobiolaging.2013.09.028

Juan, F., Eduard, V., Daniel, A., María, C. I., María-Belén, S. S., Isabel, S., et al. (2015). Cerebrospinal fluid $\beta$-amyloid and phospho-tau biomarker interactions affecting brain structure in preclinical Alzheimer disease. Ann. Neurol. 76, 223-230. doi: 10.1002/ana.24186
Karas, G. B., Burton, E. J., Rombouts, S. A. R. B., Schijndel, R. A. V., O’Brien, J. T., Scheltens, P. H., et al. (2003). A comprehensive study of gray matter loss in patients with Alzheimer's disease using optimized voxel-based morphometry. Neuroimage 18, 895-907. doi: 10.1016/S1053-8119(03)00041-7

Karas, G. B., Scheltens, P., Rombouts, S. A. R. B., Visser, P. J., van Schijndel, R. A., Fox, N. C., et al. (2004). Global and local gray matter loss in mild cognitive impairment and Alzheimer's disease. Neuroimage 23, 708-716. doi: 10.1016/j.neuroimage.2004.07.006

Kuperberg, G. R., Broome, M. R., McGuire, P. K., David, A. S., Eddy, M., Ozawa, F., et al. (2003). Regionally localized thinning of the cerebral cortex in schizophrenia. Arch. Gen. Psychiatry 60, 878-888. doi: 10.1001/archpsyc.60.9.878

Lee, Y. M., Chung, Y. I., Park, J. M., Lee, B. D., Moon, E., Jeong, H. J., et al. (2016). Decreased gray matter volume is associated with the subtypes of psychotic symptoms in patients with antipsychotic-naïve mild or moderate Alzheimer's disease: a voxel-based morphometry study. Psychiatry Res. Neuroimaging 249, 45-51. doi: 10.1016/j.pscychresns.2015.12.002

Lerch, J. P., Pruessner, J. C., Alex, Z., Harald, H., Teipel, S. J., and Evans, A. C. (2005). Focal decline of cortical thickness in Alzheimer's disease identified by computational neuroanatomy. Cereb. Cortex 15, 995-1001. doi: 10.1093/cercor/bhh200

Li, K., Luo, X., Zeng, Q., Huang, P., Shen, Z., Xu, X., et al. (2019). Gray matter structural covariance networks changes along the Alzheimer's disease continuum. NeuroImage Clin. 23:101828. doi: 10.1016/j.nicl.2019.101828

Li, R., Rui, G., Chen, W., Li, S., Schulz, P. E., and Zhang, Y. (2018). Early detection of Alzheimer's disease using noninvasive near-infrared spectroscopy. Front. Aging Neurosci. 10:366. doi: 10.3389/fnagi.2018.00366

Li, R., Rui, G., Zhao, C., Wang, C., Fang, F., and Zhang, Y. (2019). Functional network alterations in patients with amnestic mild cognitive impairment characterized using functional near-infrared spectroscopy. IEEE Trans. Neur. Syst. Rehabil. Eng. 28, 123-132. doi: 10.1109/TNSRE.2019.2956464

Liu, J., Wu, F., and Wang, J. (2020). Enhancing the feature representation of multimodal MRI data by combining multi-view information for MCI classification. Neurocomputing 400, 322-332. doi: 10.1016/j.neucom.2020.03.006

Luders, E., Thompson, P. M., Narr, K. L., Toga, A. W., Jancke, L., and Gaser, C. (2006). A curvature-based approach to estimate local gyrification on the cortical surface. Neuroimage 29, 1224-1230. doi: 10.1016/j.neuroimage.2005.08.049

Lui, L. M., Wong, T. W., Zeng, W., Gu, X. F., Thompson, P. M., Chan, T. F., et al. (2010). Detection of shape deformities using yamabe flow and Beltrami coefficients. Inverse Probl. Imag. 4, 311-333. doi: 10.3934/ipi.2010.4.311

Manuello, J., Nani, A., Premi, E., Borroni, B., Costa, T., Tatu, K., et al. (2017). The pathoconnectivity profile of Alzheimer's disease: a morphometric coalteration network analysis. Front. Neurol. 8:739. doi: 10.3389/fneur.2017.00739

Messe, A. (2019). Parcellation influence on the connectivity-based structurefunction relationship in the human brain. Hum. Brain Mapp. 41, 1167-1180. doi: 10.1002/hbm.24866

Moller, C., Vrenken, H., Jiskoot, L., Versteeg, A., Barkhof, F., Scheltens, P., et al. (2013). Different patterns of gray matter atrophy in earlyand late-onset Alzheimer's disease. Neurobiol. Aging 34, 2014-2022. doi: 10.1016/j.neurobiolaging.2013.02.013

Ottoy, J., Niemantsverdriet, E., Verhaeghe, J., Roeck, E. D., Struyfs, H., Somers, C., et al. (2019). Association of short-term cognitive decline and MCI-to-AD dementia conversion with CSF, MRI, amyloid- and ${ }^{18}$ F-FDG-PET imaging. NeuroImage Clin. 22:101771. doi: 10.1016/j.nicl.2019.101771

Petersen, R. C., Aisen, P. S., Beckett, L. A., Donohue, M. C., Gamst, A. C., Harvey, D. J., et al. (2010). Alzheimer's disease neuroimaging initiative (ADNI): clinical characterization. Neurology 74, 201-209. doi: 10.1212/WNL.0b013e31 $81 \mathrm{cb} 3 \mathrm{e} 25$

Phillips, J. S., Da Re, F., Dratch, L., Xie, S. X., Irwin, D. J., McMillan, C. T., et al. (2018). Neocortical origin and progression of gray matter atrophy in nonamnestic Alzheimer's disease. Neurobiol. Aging 63, 75-87. doi: 10.1016/j.neurobiolaging.2017.11.008

Qian, W., Schweizer, T. A., Churchill, N. W., Millikin, C. P., Ismail, Z., Smith, E. E., et al. (2019). Gray matter changes associated with the development of delusions in Alzheimer disease. Am. J. Geriatr. Psychiatry 27, 490-498. doi: 10.1016/j.jagp.2018.09.016

Righart, R., Schmidt, P., Dahnke, R., Biberacher, V., Beer, A., Buck, D., et al. (2017). Volume versus surface-based cortical thickness measurements: a 
comparative study with healthy controls and multiple sclerosis patients. PLoS ONE 12:e0179590. doi: 10.1371/journal.pone.0179590

Risacher, S. L., Saykin, A. J., West, J. D., Shen, L., Firpi, H. A., and McDonald, B. C. (2009). Baseline MRI predictors of conversion from MCI to probable $\mathrm{AD}$ in the ADNI cohort. Curr. Alzheimer Res. 6, 347-361. doi: $10.2174 / 156720509788929273$

Romerogarcia, R., Atienza, M., and Cantero, J. L. (2016). Different scales of cortical organization are selectively targeted in the progression to Alzheimer's disease. Int. J. Neural Syst. 26:1650003. doi: 10.1142/S0129065716500039

Rosas, H. D., Salat, D. H., Lee, S. Y., Zaleta, A. K., Pappu, V., Fischl, B., et al. (2008). Cerebral cortex and the clinical expression of Huntington's disease: complexity and heterogeneity. Brain 131, 1057-1068. doi: 10.1093/brain/awn025

Sailer, M., Fischl, B., Salat, D., Tempelmann, C., Schonfeld, M. A., Busa, E., et al. (2003). Focal thinning of the cerebral cortex in multiple sclerosis. Brain 126, 1734-1744. doi: 10.1093/brain/awg175

Sowell, E. R., Peterson, B. S., Kan, E., Woods, R. P., Yoshii, J., Bansal, R., et al. (2007). Sex differences in cortical thickness mapped in 176 healthy individuals between 7 and 87 years of age. Cerebral Cortex 17, 1550-1560. doi: 10.1093/cercor/bhl066

Sowell, E. R., Thompson, P. M., Leonard, C. M., Welcome, S. E., Kan, E., and Toga, A. W. (2004). Longitudinal mapping of cortical thickness and brain growth in normal children. J. Neurosci. 24, 8223-8231. doi: 10.1523/JNEUROSCI.1798-04.2004

Thompson, P. M., Hayashi, K. M., Greig, D. Z., Janke, A. L., Rose, S. E., James, S., et al. (2003). Dynamics of gray matter loss in Alzheimer's disease. J. Neurosci. 23, 994-1005. doi: 10.1523/JNEUROSCI.23-03-00994.2003

Thompson, P. M., Hayashi, K. M., Sowell, E. R., Gogtay, N., Giedd, J. N., Rapoport, J. L., et al. (2004). Mapping cortical change in Alzheimer's disease, brain development, and schizophrenia. Neuroimage 23, 2-18. doi: 10.1016/j.neuroimage.2004.07.071

Tijms, B. M., Kate, M., Gouw, A. A., Borta, A., Verfaillie, S., Teunissen, C. E., et al. (2018). Gray matter networks and clinical progression in subjects with predementia Alzheimer's disease. Neurobiol. Aging 61, 75-81. doi: 10.1016/j.neurobiolaging.2017.09.011

Tijms, B. M., Möller, C., Vrenken, H., Wink, A. M., De, H. W., Wm, V. D. F., et al. (2013). Single-subject grey matter graphs in Alzheimer's disease. PLoS ONE 8:e58921. doi: 10.1371/annotation/6a2e6405-ce1d-49e0-a88c-0017c680d597
Vivek, S., Howard, C., Lerch, J. P., Evans, A. C., Dorr, A. E., and Noor Jehan, K. (2006). Spatial patterns of cortical thinning in mild cognitive impairment and Alzheimer's disease. Brain 129, 2885-2893. doi: 10.1093/brain/awl256

Wang, L., Zou, L., Chen, Q., Su, L., Xu, J., Zhao, R., et al. (2020). Gray matter structural network disruptions in survivors of acute lymphoblastic leukemia with chemotherapy treatment. Acad. Radiol. 27, 27-34. doi: 10.1016/j.acra.2019.04.010

Wang, Y., Xu, C., Park, J. H., Lee, S., Stern, Y., Yoo, S., et al. (2019). Diagnosis and prognosis of Alzheimer's disease using brain morphometry and white matter connectomes. Neuroimage Clin. 23:101859. doi: 10.1016/j.nicl.2019.101859

Wee, C. Y., Liu, C., Lee, A., Poh, J. S., and Ji, H. (2019). Cortical graph neural network for $\mathrm{AD}$ and $\mathrm{MCI}$ diagnosis and transfer learning across populations. NeuroImage Clin. 23, 101929. doi: 10.1016/j.nicl.2019.101929

Wu, Z., Peng, Y., Selvaraj, S., Schulz, P. E., and Zhang, Y. (2020). Development of brain structural networks over age 8: a preliminary study based on diffusion weighted imaging. Front. Aging Neurosci. 12:61. doi: 10.3389/fnagi.2020.00061

Wu, Z., Xu, D., Potter, T., and Zhang, Y. (2019). Effects of brain parcellation on the characterization of topological deterioration in Alzheimer's disease. Front. Aging Neurosci. 11:113. doi: 10.3389/fnagi.2019.00113

Yong, H., Zhang, C., and Alan, E. (2008). Structural insights into aberrant topological patterns of large-scale cortical networks in Alzheimer's disease. J. Neurosci. 28, 4756-4766. doi: 10.1523/JNEUROSCI.0141-08.2008

Yotter, R. A., Nenadic, I., Ziegler, G., Thompson, P. M., and Gaser, C. (2011). Local cortical surface complexity maps from spherical harmonic reconstructions. Neuroimage 56, 961-973. doi: 10.1016/j.neuroimage.2011.02.007

Conflict of Interest: The authors declare that the research was conducted in the absence of any commercial or financial relationships that could be construed as a potential conflict of interest.

Copyright (c) 2021 Wu, Peng, Hong and Zhang. This is an open-access article distributed under the terms of the Creative Commons Attribution License (CC BY). The use, distribution or reproduction in other forums is permitted, provided the original author(s) and the copyright owner(s) are credited and that the original publication in this journal is cited, in accordance with accepted academic practice. No use, distribution or reproduction is permitted which does not comply with these terms. 